\title{
Statistical Theory of Knowledge as Instrument of Management of Innovative Potential of a Scientist
}

\author{
S.A. Filin ${ }^{1, *}$, A.ZH. Yakushev ${ }^{1}$ \\ ${ }^{1}$ Department of Management, Plekhanov Russian Economic Universit, Stremyanny Lane, 36, \\ Moscow, Russia \\ *Corresponding author
}

Keywords: Scientist, Researcher, Inventor, Innovation, Innovation Potential, Statistical Theory of Knowledge.

Abstract: At present, questions of the motivation of scientific and innovative activities of scientists and researchers are highly relevant because each state seeks to increase the level of innovation in enterprises, which in turn will ensure high growth rates of the economy, primarily due to high technologies, innovative products and services. The high level of economic development and the scientific and technological success of many corporations and entire countries is the result of an optimized policy for managing their innovation potential. The development of such a policy should be supported by logical and mathematical models. A logical and mathematical models of innovation activity in an organization, based on the statistical theory of knowledge, that allows a quantitative assessment of the possibility of increasing the innovative potential of a scientist-researcher during the various stages of the innovation process are offered.

\section{Introduction}

In a narrow sense, the concept of a "new" knowledge-based economy is based on a technocratic approach, scientific and technological progress and global financial capital, concentrated in economically developed countries, and focuses attention on the technological and organizational and economic aspects of innovation. economy. It is based on high-tech information and cognitive activities.

Therefore, at present only the increase in the productivity of workers who are not engaged in manual labor is of key importance, in the sphere where knowledge is the object and result of labor, and the use of material resources is of an auxiliary nature. Of particular importance is the increase in the productivity of scientists, researchers, innovators, inventors and specialists (consultants) on the introduction of innovations. In this area, instrumental knowledge acquires a special value, with the help of which the capital of scientific knowledge is transformed into applied, commercialized knowledge - innovations. An example of such a capital of knowledge, which plays the role of tools in the production of knowledge capital with a high market value, can serve as scientific and design schools.

At the microlevel depending on the development strategy of the organization its innovative potential can also have various quality. Most the industrial enterprises get and introduce innovations 
at this or that extent of their adaptation while the enterprises of the innovative sector of economy develop them. The management of the organization needs to provide employees with the opportunity to receive the timely and high-quality information and knowledge necessary for their activities, contributing to the realization of the scientific potential and the expanded reproduction of the human capital such as modern scientist-researchers (inventors).

\section{The Logical Model of the Statistical Theory of Knowledge}

Information and knowledge may be characterized by interrelated indicators - quantity and quality, the actual values of which may be known or not known, but which are characterized by uncertainty expressed through entropy. In particular, according to G. Castler [1], information qualitatively (semantic way) - eliminated uncertainty in achieving the goal, and quantitatively (based on the expression for the uncertainty of C. Shannon [2]), becomes such (synthesized) in the result of memorizing the choice of randomness.

At the same time, using only information, no matter how high-quality and in whatever quantity it is, once ceases to ensure the transition of the control object to a more stable (with less entropy) state in the future, that is there is a certain limit for information, after which knowledge must be in order for a control object to become more stable in the future in order for a qualitative leap to occur.

In turn, the general uncertainty refers to the possibility of transition of a control object from a certain state in the present to a more stable (with less entropy) state in the future with less ambiguity in the control object quantitative and qualitative characteristics through the use of control by the subject in the process of achieving goals information and knowledge, providing increased certainty in the situation with the inevitable choice when making management decisions $[3,6]$.

In turn, the general uncertainty refers to the possibility of transition of a control object from a certain state in the present to a more stable (with less entropy) state in the future with less ambiguity in the control object quantitative and qualitative characteristics through the use of control by the subject in the process of achieving goals information and knowledge, providing increased certainty in the situation with the inevitable choice when making management decisions [3].

According to V.V. Kovalev knowledge is the application of "information to information". As a result, within the framework of the statistical theory, the formation of a logical model of knowledge formation is presented in Figure 1.

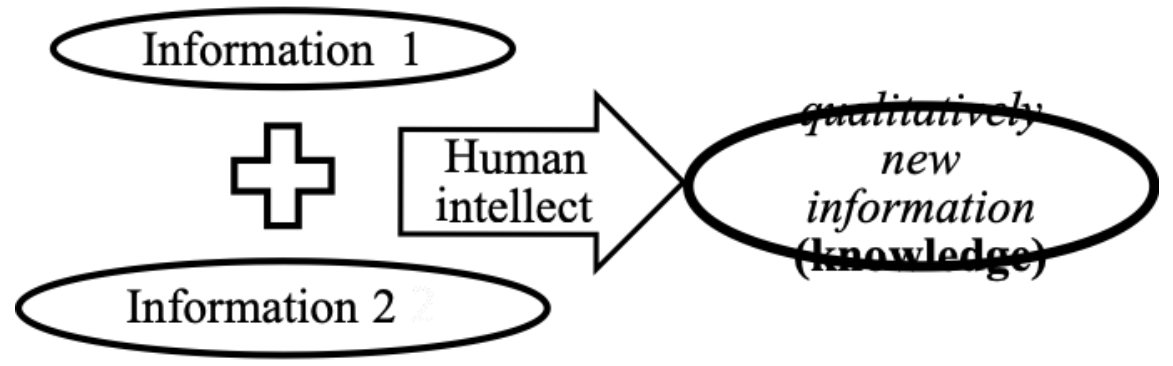

Figure 1. The Logical Model of Knowledge Formation.

According to [4], “... information and knowledge, these factors, which are specific in their nature and forms of participation in the production process, take the form of intellectual capital within firms". As a result, the formation of intellectual capital (invention) is presented in Figure 2. 


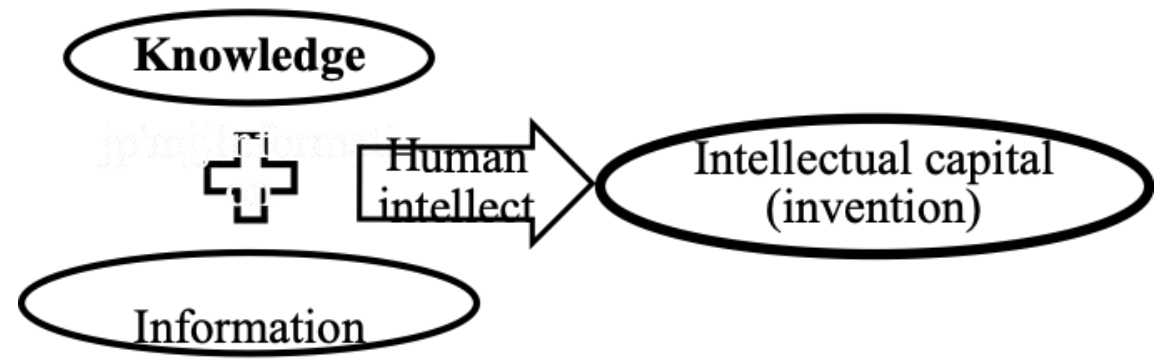

Figure 2. The Logical Model of the Formation of the Invention.

The invention can be assessed and reflected in the financial statements of the organization as a tangible asset, or sold under a license agreement in the form of a patent for an invention or knowhow. In this case, information and knowledge is capitalized into intellectual capital. If information and knowledge used to create an invention have previously been evaluated and reflected in the organization's financial statements as a tangible asset, then the "information capital" and / or "knowledge capital" will be used in the logical model.

\section{Mathematical Model of the Statistical Theory of Knowledge}

By analogy with K. Shannon's statistical information theory [2]), a part of the entropy value as a physical variable responsible for the realization of an object or process (uncertainty, eliminated, for example, by the fact that an innovative project is being created for creating an innovative product) is both semantic and quantitative measures of information and knowledge about this object or process. The quality (meaning and value) of information and knowledge is often determined by the amount of their contribution to reducing the uncertainty of choice (reducing entropy) when making management decisions. Information and knowledge can be: valuable (useful) (when choosing the right management decision to reduce uncertainty (entropy)); useless (the level of uncertainty does not change) and false and unscientific (if you choose the wrong management decision, increase uncertainty). An increase in entropy is always accompanied by a decrease in the quality of information and knowledge, and, conversely, an increase in their quality is accompanied by a decrease in the entropy of the system. However, the quality of information and knowledge is not limited to solving the problem of reducing uncertainty when choosing the optimal management decision, since the possibility of reducing uncertainty (entropy) is realized within the framework of the economic subject, and the management decision made is intended both for internal use and is a kind of significant event for external economic actors and / or potential investors (for example, the decision to implement an innovative project on a firm scale or a program on a state scale) [5].

Shannon K. [2] in the statistical information theory introduced the entropy measure of the amount of information (it is assumed that we are talking about all its diversity), which is defined as a measure of uncertainty when choosing from a variety of alternatives possible management decisions in the form:

$$
s=-\sum_{i=1}^{m} p_{i} \log p_{i}
$$

where pi - the probability of the $\mathrm{i}$ - outcome, $\mathrm{m}$ is the number of possible outcomes. 
Then, according to the picture (1) of the logical model, taking into account the synergistic effect of the interaction of information 1 with information 2 provided by human intelligence, the entropy measure of the amount of knowledge (knowledge capital) (skkn) will be defined as:

$$
S_{k k n}=-\left(\sum_{i=1}^{m} p_{i} \log p_{i}\right)^{n}
$$

where $\mathrm{n}>2$.

According to Figure (2) of the logical model, taking into account the synergistic effect of the interaction of knowledge with information provided by human intelligence, the entropy measure of the amount of intellectual capital (sink) (inventions) will be defined as:

$$
S_{\text {ink }}=-\sum_{i=1}^{m} p_{i} \log p_{i}\left(\sum_{i=1}^{m} p_{i} \log p_{i}\right)^{n}=\left(\sum_{i=1}^{m} p_{i} \log p_{i}\right)^{n+1} .
$$

Expressions (1-3) can be represented in the form of non-summable variants, for example, for information in which there are messages, data, etc., containing incomparable features:

a) in the statistical theory of knowledge proposed by the authors, the mathematical quantitative definition of entropy is described using uncertainty. In this theory, the main goal is the transfer of knowledge (knowledge capital), and its implementation assumes the existence of identical sets of knowledge capital (specified and known at the input and output of the knowledge transfer system (knowledge capital)) at the transmitting and receiving sides;

b) probabilities and uncertainty in expressions (1-3) are considered in relation to a specific knowledge (knowledge capital) about the coded with the participation of these sets of possible messages about knowledge (knowledge capital).

Strategic management of the organization and innovation imply, as a rule, mandatory variability in the development of management decisions. Potentially possible alternatives and the choice of them suggest a certain probability of their implementation, the value of which depends on the information security and knowledge (on the amount of prior information and knowledge), as well as the availability of criteria for the optimality of this choice, determined by the investment and innovation ideology, strategy and company policy (university).

The statistical theory of knowledge is based on the principle of the possibility of increasing the likelihood of implementing at the point of an unstable state in an organization the optimal possible scenario of their development by using the subject of management of a company (university) in a situation with the inevitable choice in making management decisions of quality information and development knowledge state of the object, allowing to reduce uncertainty (reduce entropy) and increase the likelihood of realization by means of management decisions optimal development scenario.

The ultimate economic goal of the process of capitalization of knowledge (the transformation of an intellectual product into additional capital) within the framework of the statistical theory of knowledge is their commercialization and the possibility of subsequent use as a production factor necessary to achieve the organization's global competitive advantage and ensuring the multiplicative effect of their economic growth.

Limitations of the statistical theory of knowledge and the corresponding mathematical model:

a) information and knowledge can only be attributed to those that reveal the object under study from a new, previously unknown side, i.e., which reduce the uncertainty (reduce entropy) associated with the object or choice when making the appropriate management decision on the patenting of an 
invention or the design of an invention in the form of know-how and implementation based on the invention of innovation;

b) the above expressions (1-3) mainly include an assessment of the quantity of information (information capital) and knowledge (knowledge capital), and their quality (value (utility)), including the meaning (content) or meaning, is not considered;

c) the subjectivity of information and knowledge and their variability over time are not taken into account;

d) since there are no two absolutely identical individuals and groups, it is problematic to use statistical weight as a measure of the amount of information and knowledge that they have. Even if the statistical weight of information and knowledge available to an "individual" is taken for a certain amount, for example " 1 ", the system "collective" will not be the sum of the statistical weights of information and knowledge that make up its individuals, since the quantity and quality of information and knowledge in the system The "team" can be implemented in different ways;

e) the properties of information and knowledge are not reflected in the following aspects: material (their recording and storage on relevant media and the possibility of reflection in accounting (capitalization)); casual (their role in cause-effect relationships); epistemological (information and knowledge as a means of knowledge); reflection-theoretic (their role in the mapping processes); communicative (informational and cognitive communication in the system); semiotic (ways of reflecting them in a certain sign system); axiological (their value for a selfgoverning system), etc.

\section{Conclusions}

a) in the statistical theory of knowledge proposed by the authors, the mathematical quantitative definition of entropy is described using uncertainty. In this theory, the main goal is the transfer of knowledge (knowledge capital), and its implementation assumes the existence of identical sets of knowledge capital (specified and known at the input and output of the knowledge transfer system (knowledge capital)) at the transmitting and receiving sides;

b) probabilities and uncertainty in expressions (5-8) are considered in relation to a specific knowledge (knowledge capital) about the coded with the participation of these sets of possible messages about knowledge (knowledge capital).

Strategic management of the organization and innovation imply, as a rule, mandatory variability in the development of management decisions. Potentially possible alternatives and the choice of them suggest a certain probability of their implementation, the value of which depends on the information security and knowledge (on the amount of prior information and knowledge), as well as the availability of criteria for the optimality of this choice, determined by the investment and innovation ideology, strategy and company policy (university).

The statistical theory of knowledge is based on the principle of the possibility of increasing the likelihood of implementing at the point of an unstable state in an organization the optimal possible scenario of their development by using the subject of management of a company (university) in a situation with the inevitable choice in making management decisions of quality information and development knowledge state of the object, allowing to reduce uncertainty (reduce entropy) and increase the likelihood of realization by means of management decisions optimal development scenario.

The ultimate economic goal of the process of capitalization of knowledge (the transformation of an intellectual product into additional capital) within the framework of the statistical theory of knowledge is their commercialization and the possibility of subsequent use as a production factor 
necessary to achieve the organization's global competitive advantage and ensuring the multiplicative effect of their economic growth.

Limitations of the statistical theory of knowledge and the corresponding mathematical model:

a) information and knowledge can only be attributed to those that reveal the object under study from a new, previously unknown side, i.e., which reduce the uncertainty (reduce entropy) associated with the object or choice when making the appropriate management decision on the patenting of an invention or the design of an invention in the form of know-how and implementation based on the invention of innovation;

b) the above expressions (1-3) mainly include an assessment of the quantity of information (information capital) and knowledge (knowledge capital), and their quality (value (utility)), including the meaning (content) or meaning, is not considered;

c) the subjectivity of information and knowledge and their variability over time are not taken into account;

d) since there are no two absolutely identical individuals and groups, it is problematic to use statistical weight as a measure of the amount of information and knowledge that they have. Even if the statistical weight of information and knowledge available to an "individual" is taken for a certain amount, for example " 1 ", the system "collective" will not be the sum of the statistical weights of information and knowledge that make up its individuals, since the quantity and quality of information and knowledge in the system The "team" can be implemented in different ways;

e) the properties of information and knowledge are not reflected in the following aspects: material (their recording and storage on relevant media and the possibility of reflection in accounting (capitalization)); casual (their role in cause-effect relationships); epistemological (information and knowledge as a means of knowledge); reflection-theoretic (their role in the mapping processes); communicative (informational and cognitive communication in the system); semiotic (ways of reflecting them in a certain sign system); axiological (their value for a selfgoverning system), etc.

\section{References}

[1] Quastler, H. (1964) The emergence of a biological organization. New Haven and London: Yale University Press, 83 p.

[2] Shannon, Claude E. (1949) Communication Theory of Secrecy Systems, Bell System Technical Journal, 28, 656-715.

[3] Filin S.A. (2010) Theoretical foundations and methodology of strategic management of innovative development. Tula: Publishing House of TSU, $433 \mathrm{p}$.

[4] Inozemtsev V.L. (1998) Outside the economic society, Moscow: Academia. The science, 640 p.

[5] Lafta J.K. Management decisions: studies. allowance. M .: Blagovest-B, 2004, 304 p.

[6] Khachaturyan M.V., Klicheva E.V., Velikorossov V. V. (2019) Digital Mechanisms of Development of Possessory Risk Management Systems under New Economic Conditions. Proceedings of the 2019 International Conference on Politics, Economics and Management (ICPEM 2019). Lecture Notes in Economics, Management, and Social Sciences. Clausius Scientific Press, Vol. 5. P. 6-10. 